works, the changes which have caused such trouble in the northern hemisphere recently (and indeed those which are causing trouble in Australia now) might have been predicted in time for effective action to be taken.

As Kukla and Kukla point out ( $\mathrm{Sci}$ ence, 183, 709; 1974), the location and duration of snow and pack ice fields are the most important seasonal factors which affect the Earth's heat balance. Because of the heat required for melting snow and ice, peak air temperatures in summer at mid-latitudes are delayed after the peak insolation. In the northern hemisphere, permanent ice covers some $10 \times 10^{6} \mathrm{~km}^{2}$, and seasonal ice and snow covers a further $50 \times 10^{6} \mathrm{~km}^{2}$; in the south, the corresponding figures are $14 \times 10^{6} \mathrm{~km}^{2}$ and $20 \times 10^{6} \mathrm{~km}^{2}$. The 'temporary' cover in the north is greater, of course, because of the presence of continents on which the snow can lie. Satellite mapping provides the only reliable means of monitoring the changing ice patterns over a whole hemisphere.

These studies are facilitated by dividing the year into four snow cover (SC) seasons. SC summer is the part of the year when less than $15 \times 10^{6} \mathrm{~km}^{2}$ of the northern hemisphere are covered; $\mathrm{SC}$ winter the period when more than $55 \times 10^{6} \mathrm{~km}^{2}$ are covered. During the past $7 \mathrm{yr}$, SC winter and SC summer have been about the same length, averaging 80 to $90 \mathrm{~d}$. But SC spring was, on average, about 7 weeks longer than $\mathrm{SC}$ fall. And the date on which SC fall and winter started were earlier in each of the successive years 1971, 1972 and 1973. In 1971, the change was dramatic, with SC fall starting 3 weeks earlier than in any of the 4 preceding years.

During the same period, there was a gradual increase in snow and ice cover found during the October/November period. For the entire $7 \mathrm{yr}$ of this study, the average annual coverage was $34.9 \times$ $10^{6} \mathrm{~km}^{2}$; in 1971 alone this increased by $12 \%$ and since 1971 the average has fluctuated about a new mean of $36.9 \times$ $10^{6} \mathrm{~km}^{2}$. On November 1,1973 , the cover was $36.7 \times 10^{6} \mathrm{~km}^{2}$. So the period since 1967 divides naturally into two distinctly different sections.

Kukla and Kukla discuss at some length the possible sources of error in their study, and argue a convincing case that the effect they report is real. "There is no doubt", they say, "that the surface heat exchange in 1971 must have dropped

\title{
Synthesis in vitro of silkworm ecdysone
}

from our Insect Physiology Correspondent

Twelve months ago some of the queries that have arisen concerning the site of synthesis of ecdysone in insects were discussed (see Nature, 242, 86; 1973). Briefly, the problem has been that, in spite of the conclusive experimental evidence (provided originally by Fukuda) that the moulting factor of insects is produced by the prothoracic glands, it has not proved possible to get convincing proof that the product ecdysone as isolated by Karlson and Butenandt is synthesised by these glands. Only the most minute amounts of ecdysone from the prothoracic glands have been extracted, and glands maintained in vitro produced only insignificant amounts of the hormone.

A group of Japanese workers led by Chino (Science, 183, 529; 1974) have now reinvestigated this problem and have found, as earlier workers had done, that the isolated prothoracic glands of the silkworm Bombyx maintained in the culture media of Grace or of Wyatt produced only minute amounts of ecdysone (less than $5 \mathrm{ng}$ per pair of glands). Unchanged haemolymph blackens and is an unsatisfactory medium; but by isolating the haemolymph proteins on a Sephadex column and suspending these in Wyatt's culture medium and saline Chino and his colleagues attained a far more satisfactory "haemolymph medium". Arguing that the rich tracheal supply of the glands indicated a high oxygen requirement for synthesis, they exposed the gland pairs, each pair in a single drop culture, to oxygen instead of air. By these combined procedures the hormone produced per gland pair increased in amount from $5 \mathrm{ng}$ to $120 \mathrm{ng}$.

It is well known that the insect requires cholesterol for ecdysone synthesis. The authors therefore concluded that the lipoproteins of the haemolymph are likely to be the essential component so they then purified the two chief lipoproteins I and II from the haemolymph and prepared a culture medium by addition of these to Wyatt's medium-and this proved even more effective than the haemolymph medium. The hormone synthesised by the glandsi is at once liberated to the medium; in agreement. with earlier observations, almost no ecdysone can be extracted from the glands themselves. Thin layer and gas chromatography established that the hormone produced by the glands is solely $\alpha$ ecdysone; as had already been suspected the more active and more polar product, $\beta$ ecdysone must be formed from $\alpha$ ecdysone elsewhere in the body. Chino et al. report that another research team in the laboratory of Gilbert, working independently, has obtained similar results with the prothoracic glands of the tobacco hornworm Manduca sexta (Proc. natn. Acad. Sci. $U . S . A .$, in the press). markedly". It is still too early to say definitely that this caused the change in global circulation which has, in recent months, been the subject of a great deal of discussion both in these pages and elsewhere; but it may well be significant that the changes in ice cover occurred before these changes in circulation became apparent. It seems plausible, at least, to argue that the observed changes in mean temperature and other parameters during the past $30 \mathrm{yr}$ eventually produced the increased snow cover which has in some way disturbed the atmospheric circulation.

\section{Superfluid ${ }^{3} \mathrm{He}$ confirmed}

from our

Condensed Matter Correspondent

ThE report in a recent issue of Physical Review Letters $(32,141 ; 1974)$ by Kojima, Paulson and Wheatley of the University of California at San Diego that they have observed the propagation of fourth sound in the two newly discovered phases of liquid ${ }^{3} \mathrm{He}$ amounts to the first unequivocal evidence that both of these new phases are superfluids.

It has long been known that liquid ${ }^{4} \mathrm{He}$ is superfluid below its transition temperature at about $2 \mathrm{~K}$ but, until 1972 , it was part of the conventional wisdom that the other isotope, ${ }^{3} \mathrm{He}$, was not a superfluid. This was mainly because of the theoretical impossibility that liquid ${ }^{3} \mathrm{He}$, whose atoms contain an odd number of fundamental particles and are called fermions, could become superfluid in the same manner as liquid ${ }^{4} \mathrm{He}$ by undergoing a BoseEinstein condensation; this phenomenon can only occur in assemblies whose components, bosons, contain an even number of fundamental particles. It was realised, however, that the electrons (also fermions) in a superconductor, at that time the only other known superfluid, acquired their superfluidity in a somewhat different way from ${ }^{4} \mathrm{He}$; and it had been suggested that a transition analogous to the superconducting transition in metals might occur in liquid ${ }^{3} \mathrm{He}$ at a sufficiently low temperature, leading to some sort of superfluid state.

The first indication that this suggestion might indeed have been correct came some fifteen years later when Osheroff, Richardson and Lee of Cornell University discovered that two quite separate phase transitions occurred in a cell containing a mixture of liquid and solid ${ }^{3} \mathrm{He}$ when it was cooled under pressure below $3 \mathrm{mK}$ (Phys. Rev. Lett., 28, 885; 1972). In subsequent experiments, Alvesalo, Anufriyev, Collan, Lounasmaa and Wennerström of the Technical University at Helsinki used 